\title{
Expression of EGFR in Primary Squamous Cell Carcinoma of Lung
}

\author{
Karthika Padmavathy ${ }^{1}$, Vasugi. G. A ${ }^{1 *}$, Elakkiya Ilavarasan ${ }^{1}$, Vijayalakshmi Thanasekaran ${ }^{2}$, Prathiba Duvuru ${ }^{1}$ \\ ${ }^{1}$ Department of Pathology, Sri Ramachandra Medical College and Research Institute, No.1, Ramachandra Nagar, Sri Ramachandra Nagar, Porur, \\ Chennai, Tamil Nadu 600116, India \\ ${ }^{2}$ Department of Pathology, Chest and TB, Sri Ramachandra Medical College and Research Institute, No.1, Ramachandra Nagar, Sri Ramachandra \\ Nagar, Porur, Chennai, Tamil Nadu 600116, India
}

\author{
DOI: $10.36348 /$ sjpm.2019.v04i11.008 \\ | Received: 09.11.2019 | Accepted: 17.11.2019 | Published: 25.11.2019 \\ *Corresponding author: Dr. G. A. Vasugi
}

\section{Abstract}

Background: Lung cancer is one of the leading cause of death in developing countries. Epidermal growth factor receptor (EGFR) is a transmembrane protein with cytoplasmic kinase activity that transduces important growth factor signaling from the extracellular milieu to the cell. Materials and methods: This study includes all cases of primary lung squamous cell carcinomas presented in the Department of Pathology from Jan 2011 to Dec 2013 in Sri Ramachandra Medical College. Formalin fixed paraffin embedded tissue stained with H\&E were used for routine morphology. Immunohistochemical staining was done using monoclonal anti EGFR antibody by Streptavidin biotin peroxidase complex technique. Positive cases were graded as $1+, 2+, 3+$ based on staining intensity. Results: A total of 149 cases of carcinoma lung were retrieved. Of these $26(20 \%)$ were SCC. Eighty eight percent of these were EGFR positive, of which $70 \%$ showed $3+$ positivity, $11 \%$ showed $2+$ positivity, $7 \%$ showed $1+$ positivity. Conclusion: EGFR expression appears to be significant in primary squamous cell carcinoma of the lung which aid in treating these patients with tyrosine kinase inhibitors and may improve the outcome of these patients. We conclude that EGFR marker expression is mandatory not only in lung adenocarcinomas but also in Primary squamous cell carcinoma of the lung.

Keywords: Lung cancer, Epidermal growth factor receptor (EGFR), morphology.

Copyright @ 2019: This is an open-access article distributed under the terms of the Creative Commons Attribution license which permits unrestricted use, distribution, and reproduction in any medium for non-commercial use (NonCommercial, or CC-BY-NC) provided the original author and sources are credited.

\section{INTRODUCTION}

Lung cancer is the number one cause of cancer deaths in the world, with little improvement in 5-year survival during the past half century. Five-year survival for male lung cancer patients ranges from $6 \%$ to $14 \%$ and for female patients ranges from $7 \%$ to $18 \%$. Squamous cell carcinoma (SCC) lung is the second most common subtype of non-small cell lung cancer (NSCLC). Annual deaths from lung cancer surpass the combined total deaths from the next three leading causes of cancer deaths (colon, breast, and prostate cancers) combined. Worldwide, the number of estimated deaths from lung cancer in 2011 was 1378 400 .

Epidermal Growth Factor Receptor (EGFR) mutation is a Predictive biomarker for EGFR-tyrosine kinase inhibitor therapy. The survival rate in stage IV disease is an alarming $10 \%$ with a median survival of 8 months.

Normal lung expresses low level EGFR. Overexpression of EGFR is reported in pre neoplastic conditions and in many SCC. In the FLEX et al., study patients with metastatic NSCLC cisplatin and vinorebine with cetiximab had a significantly longer survival. Patients with both SCC and adenocarcinoma were included in this trial [1].

Encouraging new treatment options like Epidermal growth factor receptor - Tyrosine Kinase inhibitors (EGFR-TKI), are found to be useful in adenocarcinomas, but their role on SCC was questionable. However, recently there are reports stating that EGFR-TKI were found to be useful in SCC. Immunohistochemical (IHC) testing of these tumors for EGFR overexpression may serve as a preliminary screening procedure. Ongoing studies suggest the possibility of developing new targeted therapies for SCC lung. With this background, we proposed to study the expression of EGFR in SCC lung in our subset of patients.

\section{MATERIALS AND METHODS}

This study includes all non small cell lung carcinomas presented in the Department of Pathology from Jan 2011 to Dec 2013. A total of 149 cases of non small cell carcinomas were identified. Institutional 
Ethics Committee approval was obtained for the study. Of these 26 cases were SCC. Poorly differentiated tumors were subjected to p63 and TTF1 immunostain. p63 positive cases were included in the study. P63 is a marker $84 \%$ sensitive and $85 \%$ specific for SCC [2].

Immunostaining of EGFR was done on 26 cases using Mouse Anti-Human EGFR Primary Antibody-Lyophilized (Novocastra) at a dilution of 1:50. A distinct membrane staining was regarded as positive staining.

A semi quantitative approach was used, to score EGFR immunostaining. At least 10 representative fields with maximum staining under high-power magnification $(\mathrm{x} 400)$ were chosen and 1000 cells were counted for each case. If the number of cells was less than 1000, then all available cells were counted and the results were expressed as mean percentage.

Positive cases were graded as $1+, 2+, 3+$ based on staining intensity. Results were analysed.

\section{RESULTS}

A total of 149 cases of carcinoma lung were retrieved. Of these $26(20 \%)$ were SCC. Poorly differentiated tumors were subjected to p63 and TTF1 immunostain. p63 positive cases were included in the study. P63 is a marker $84 \%$ sensitive and $85 \%$ specific for SCC [2].

Out of 26 cases of SCC 21(80.76\%) were males and 5(19.24) were females (Table-1). 2 cases $(7.6 \%)$ were more than 80 years. 11 cases $(42.3 \%)$ were between 61 to 80 years of age. 12 cases $(46.3 \%)$ were between the age group of 41-60 years and 1 case (3.8\%) was less than 40 years of age (Table-2).

23 SCC cases $(88.46 \%)$ out of 26 were positive for EGFR and only $3(11.5 \%)$ were negative. Of the EGFR positive cases, 18 cases $(69.29 \%)$ showed 3+ positivity, 4 cases $(10.8 \%)$ showed $2+$ positivity and 1 case $(6.8 \%)$ showed $1+$ positivity (Chart $3 \& 4)$

Table-1

\begin{tabular}{|l|l|l|l|l|}
\hline Markers & Adenocarcinoma & Squamous Cell Carcinoma & Adenosquamous & Nsclc- Nos \\
\hline TTF 1 & + & - & + & - \\
\hline P 63 & - & + & + & - \\
\hline
\end{tabular}

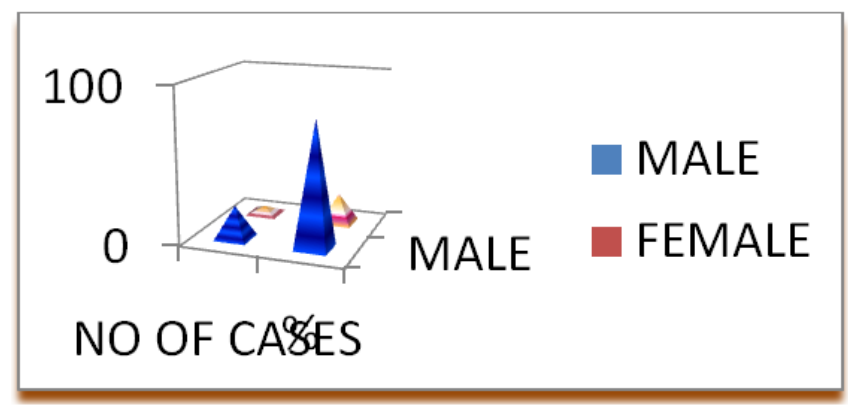

Chart-1:

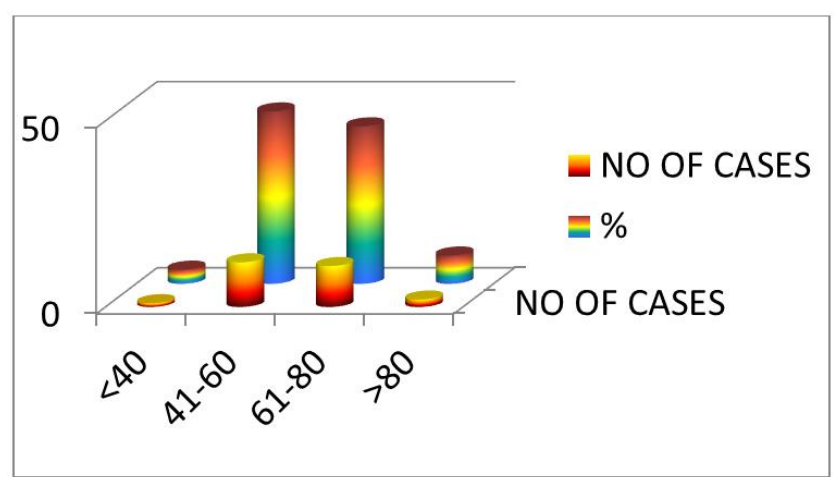

Chart-2:

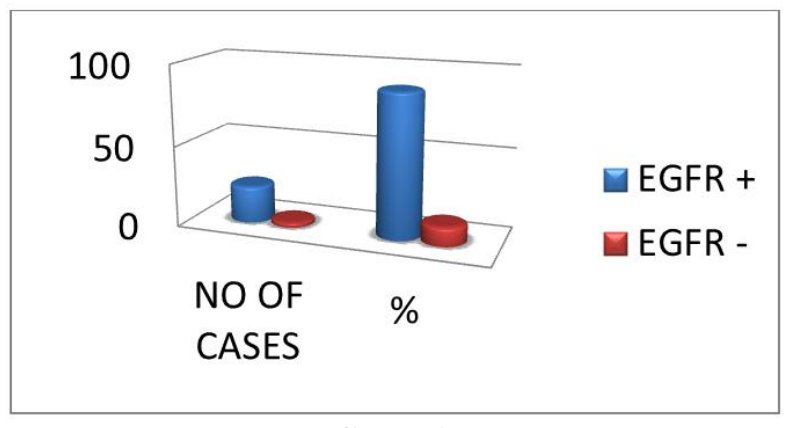

Chart-3:

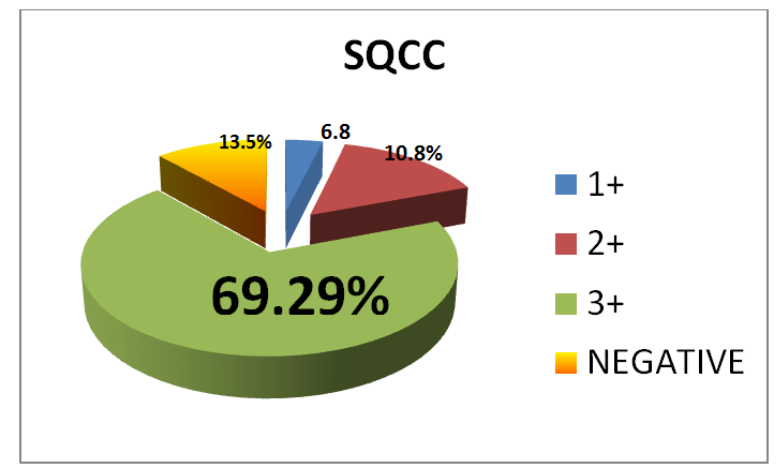

Chart-4: 


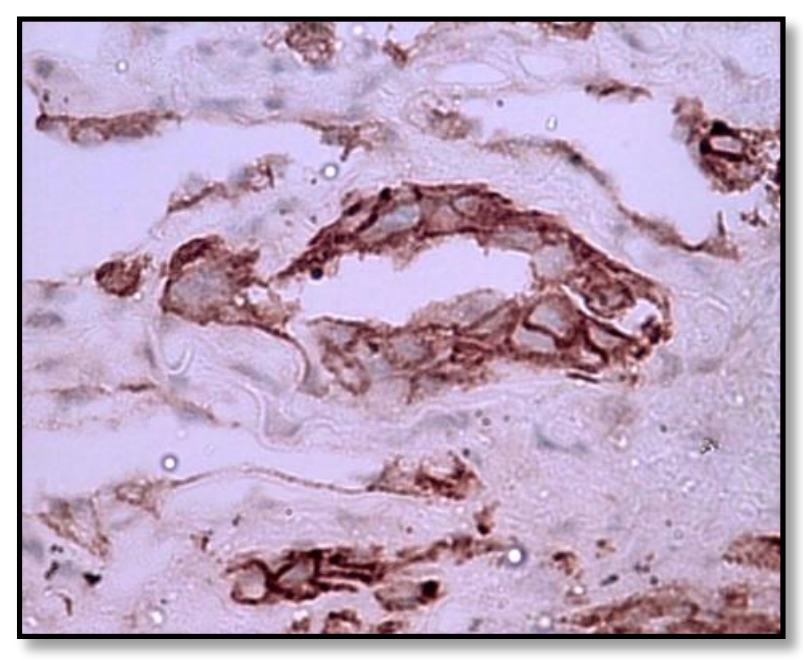

EGFR 1+

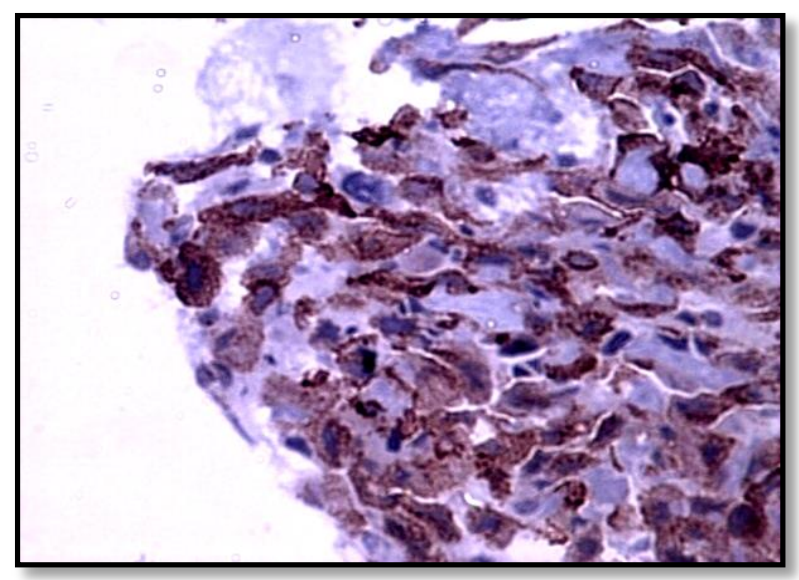

EGFR 2+

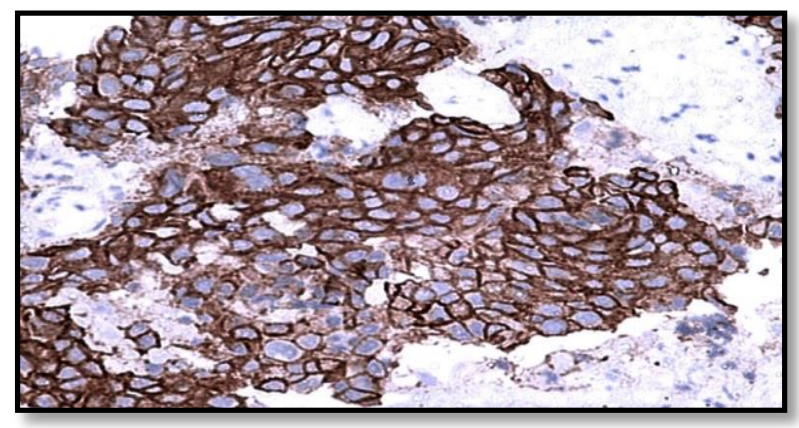

EGFR 3+

\section{DISCUSSION}

Advent of TKI targeting EGFR and its activating mutations had played an important role in management of patients with lung adenocarcinoma. The efficacy and benefit of EGFR TKIs have been established in EGFR mutation-positive ADCs, and now there are far more efficacious third-generation TKIs for ADCs progressing on first-generation TKIs $[5,6]$. The efficacy of TKIs, however, has not been well studied in EGFR mutation-positive squamous cell carcinomas (SCCs) of the lung. Clinical trials of the EGFR-TKI in patients with squamous cell carcinoma lung had modest response (10\%) which is due to lack of EGFR sensitizing alterations. However, it has been proved that monoclonal antibody therapy with cetuximab had overall significant survival rate in patients with SCC lung.

Performing EGFR testing in SCC is a matter of debate, with no uniform consensus, and guidelines vary for different organizations [7-9]. Various small series, predominantly from Eastern Asia, have reported the incidence of EGFR kinase mutations in SCC to be in the range of $2 \%-10 \%$, with the caveat that some of these studies have included adenosquamous carcinomas as well in their reports [10]. There are studies stating that the correlation between EGFR Immunostaining and their provocative response to cetuximab therapy.

Kozu Y et al., have showed that sensitivity and specificity of IHC to predict response to EGFR TKIs as $63 \%$ and $70 \%$ respectively. The high percentage of $3+$ positivity encourages further studies in this area. The above study clearly shows that squamous cell carcinoma lung is a cancer with diverse genomic causes, many of which are potentially susceptible to drug inhibition.

Lynne eldridge et al., in their study have quoted that, though EGFR mutation is uncommon in squamous cell carcinoma lung, the overall response to EGFR-TK inhibitor treatment was $17.4 \%$ and the disease control rate was $27.2 \%$ [3]. Elizabeth cooney et $a l$. , in their study, the cancer genome atlas network Comprehensive genomic characterization of squamous cell lung cancers. The above study clearly shows that squamous cell carcinoma lung is a cancer with diverse genomic causes, many of which are potentially susceptible to drug inhibition [4]. Additional ongoing studies by, Gredory J. Riely et al., will delineate the role of AFITINIB in first line when compared with first-generation EGFR-TKI as well as in patients with squamous cell lung carcinoma [5].

\section{CONCLUSION}

EGFR expression appears to be significant $(69 \%)$ in primary squamous cell carcinoma of the lung which aid in treating these patients with tyrosine kinase inhibitors and may improve the outcome of these patients. We conclude that EGFR marker expression is mandatory not only in lung adenocarcinomas but also in Primary squamous cell carcinoma of the lung.

\section{REFERENCES}

1. Gold, K. A., Wistuba, I. I., \& Kim, E. S. (2012). New strategies in squamous cell carcinoma of the lung: identification of tumor drivers to personalize therapy. Clinical Cancer Research, 18(11), 3002 3007.

2. Terry, J., Leung, S., Laskin, J., Leslie, K. O., Gown, A. M., \& Ionescu, D. N. (2010). Optimal immunohistochemical markers for distinguishing 
lung adenocarcinomas from squamous cell carcinomas in small tumor samples. The American journal of surgical pathology, 34(12), 1805-1811.

3. Lynne, E. (2012). Tarceva/erlotinib for Squamous cell carcinoma lung.

4. Cooney, E. (2012). New Potential targets discovered for treating squamous cell lung cancers. Broad Communications, Sep, 9.

5. Gredory, J. R. (2015). Afitinib in Non small cell lung carcinoma, 2012. Journal Adv Pract Oncol. 6(5): 448-455.

6. Wang, S., Tsui, S. T., Liu, C., Song, Y., \& Liu, D. (2016). EGFR C797S mutation mediates resistance to third-generation inhibitors in T790Mpositive non-small cell lung cancer. Journal of hematology \& oncology, 9(1), 59.

7. Keedy, V. L., Temin, S., Somerfield, M. R., Beasley, M. B., Johnson, D. H., McShane, L. M., ... \& Giaccone, G. (2011). American Society of Clinical Oncology provisional clinical opinion: epidermal growth factor receptor (EGFR) mutation testing for patients with advanced nonsmall-cell lung cancer considering first-line EGFR tyrosine kinase inhibitor therapy. Journal of Clinical Oncology, 29(15), 2121-2127.

8. Felip, E., Gridelli, C., Baas, P., Rosell, R., Stahel, R., \& Panel Members. (2011). Metastatic nonsmall-cell lung cancer: consensus on pathology and molecular tests, first-line, second-line, and third-line therapy: 1st ESMO Consensus Conference in Lung Cancer; Lugano 2010. Annals of Oncology, 22(7), 1507-1519.

9. Lindeman, N. I., Cagle, P. T., Beasley, M. B., Chitale, D. A., Dacic, S., Giaccone, G., ... \& Thunnissen, E. (2013). Molecular testing guideline for selection of lung cancer patients for EGFR and ALK tyrosine kinase inhibitors: guideline from the College of American Pathologists, International Association for the Study of Lung Cancer, and Association for Molecular Pathology. Arch Pathol Lab Med. 137(6):828

10. Cho, S. H., Park, L. C., Ji, J. H., Park, S., Hwang, D. W., Lee, J. Y., ... \& Park, K. (2012). Efficacy of EGFR tyrosine kinase inhibitors for nonadenocarcinoma NSCLC patients with EGFR mutation. Cancer chemotherapy and pharmacology, 70(2), 315-320. 